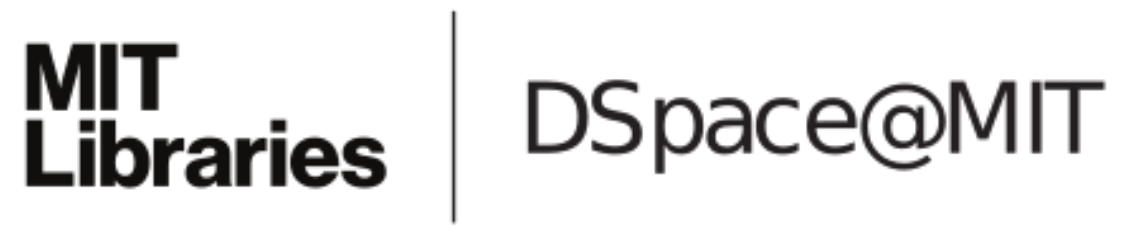

\author{
MIT Open Access Articles
}

Moving beyond two-dimensional screens to interactive
three-dimensional visualization in congenital heart disease

The MIT Faculty has made this article openly available. Please share how this access benefits you. Your story matters.

As Published: https://doi.org/10.1007/s10554-020-01853-1

Publisher: Springer Netherlands

Persistent URL: https://hdl.handle.net/1721.1/131787

Version: Author's final manuscript: final author's manuscript post peer review, without publisher's formatting or copy editing

Terms of Use: Article is made available in accordance with the publisher's policy and may be subject to US copyright law. Please refer to the publisher's site for terms of use. 


\section{Moving beyond two-dimensional screens to interactive three- dimensional visualization in congenital heart disease}

Cite this article as: John L. Byl, Rebecca Sholler, Jordan M. Gosnell, Bennett P. Samuel and Joseph J. Vettukattil, Moving beyond two-dimensional screens to interactive threedimensional visualization in congenital heart disease, The International Journal of Cardiovascular Imaging https://doi.org/10.1007/s10554-020-01853-1

This Author Accepted Manuscript is a PDF file of an unedited peer-reviewed manuscript that has been accepted for publication but has not been copyedited or corrected. The official version of record that is published in the journal is kept up to date and so may therefore differ from this version.

Terms of use and reuse: academic research for non-commercial purposes, see here for full terms. https://www.springer.com/aam-terms-v1 
John L. Byl ${ }^{1}$ MPH, RDCS (AE PE), Rebecca Sholler ${ }^{2}$, Jordan M. Gosnell ${ }^{1}$ BS, RDCS (AE PE), FASE, Bennett P. Samuel ${ }^{1}$ MHA, BSN, RN, Joseph J. Vettukattil ${ }^{1,3}$ MBBS, MD, DNB, CCST, FRCPCH, FRSM, FRCP

Moving beyond two-dimensional screens to interactive three-dimensional visualization in congenital heart disease

${ }^{1}$ Congenital Heart Center, Spectrum Health Helen DeVos Children's Hospital, Grand Rapids, MI, USA; ${ }^{2}$ Department of Mechanical Engineering, Massachusetts Institute of Technology, Cambridge, MA, USA; ${ }^{3}$ Pediatrics and Human Development, Michigan State University College of Human Medicine, Grand Rapids, MI, USA

Joseph.Vettukattil@helendevoschildrens.org; 616-267-0988

John L. Byl 0000-0002-1292-3334

Jordan M. Gosnell 0000-0002-1959-0157

Bennett P. Samuel 0000-0002-0914-9536

Joseph J. Vettukattil 0000-0002-0993-3208 


\section{Abstract}

Beginning with the discovery of X-rays to the development of three-dimensional (3D) imaging, improvements in acquisition, post-processing, and visualization have provided clinicians with detailed information for increasingly accurate medical diagnosis and clinical management. This paper highlights advances in imaging technologies for congenital heart disease (CHD), medical adoption, and future developments required to improve pre-procedural and intra-procedural guidance.

Keywords: 3D imaging, artificial intelligence, congenital heart disease, interactive 3D visualization 


\section{Introduction}

Diagnostic imaging has played a pivotal role in the medical field since 1895 when Wilhelm Röntgen discovered X-rays. Technological advances led to fluoroscopy becoming available in 1920 for radiologists and interventional cardiologists to make improved diagnoses and perform interventions of increasing complexity [1]. The introduction of ultrasound in 1956, computed tomography (CT) in 1972, and magnetic resonance (MR) imaging in 1977 transformed the medical field. Unlike X-ray and CT, MR does not use ionizing radiation for image acquisition, and soft tissue sensitivity is found to be greater than that of CT [2]. Subsequent improvements in imaging technologies now allow higher resolution, and significant increases in computing power enable visualization of both soft tissue and bone as well as faster detection of abnormalities [35]. Fast-forward several decades; we now have CT, MR and ultrasound-based 3D imaging, 3D rendering, and full-volume imaging. These technologies are providing clearer image studies of congenital heart disease (CHD). Moreover, advanced processing of individual modalities can be achieved for virtual visualization and 3D printing of complex CHD. Initially segmentation was limited to CT and MR and is now feasible with ultrasound [6]. The integration of multiple imaging modalities has been demonstrated to improve the accuracy of hybrid 3D models over single modality models $[7,8]$. The advent of augmented and virtual reality in medical imaging is transforming workflows for clinical decision-making as well as enhancing education of patients/family members and medical professionals. These new advances and innovations in medical imaging are pushing the limits on how physicians and other professionals can utilize the various imaging datasets [8], which are particularly important for the care and management of children and adults with CHD. 


\section{Current State of Imaging}

The standard-of-care imaging modalities for congenital heart disease include CT, MR, and ultrasound. Post-processing of the imaging datasets can be performed on a picture archiving and communication system (PACS). Historically, PACS vendors have provided inconsistent tools to functionally allow 3D assessment or simultaneous viewing of multiple imaging modalities, thereby limiting full anatomic evaluation [9]. Companies currently supporting PACS that are optimized for viewing CT, MR, and other imaging modalities in 3D and 4D include but are not limited to: INFINITT Inc. (North America), GE Healthcare (United States), and Philips, Koninklijke Philips N.V. (Netherlands).

Further advances in medical imaging, namely, in multi-energy CT, cardiac magnetic resonance (CMR) and 3D echocardiogram (3DE) have made it possible for high quality 3D volumetric rendering of dynamic cardiac structures $[10,8]$. Each imaging modality has different strengths: cardiac CT is the modality of choice for visualization of extracardiac and small vascular anatomy; CMR is the gold standard for quantification of ventricular volumes and myocardial architecture; and 3DE provides the best visualization of valve morphology and intracardiac structural anomalies [3-5,8]. As such, these imaging datasets have proven to be advantageous for pre-procedural planning of surgical and interventional procedures [8]. However, cumulative exposure to radiation is a major concern in patients who have image acquisition using CT or diagnostic/interventional catheterization using fluoroscopy [11,12]. In addition, there are significant limitations as viewing $3 \mathrm{D}$ anatomy on a $2 \mathrm{D}$ screen may cause each observer to interpret it from different perspectives, leading to misinterpretation and high interobserver variability [13]. In other words, the conceptualization of three-dimensionality on a 2D visualization platform requires the physician or end user to have advanced knowledge in 3D 
spatial orientation. The advent of 3D printing and 3D visualization decreases this risk as it leaves no aspect of the spatial relationship of the cardiac structures to the imagination [14].

3D Printing. 3D printing in surgical and interventional planning in complex CHD is well recognized. Prior to 2014, CT and CMR were the only modalities used to derive 3D printing cardiac models. Printing of 3D anatomic cardiac models sourced from 3DE was shown to be feasible in 2014 [6]. Combining the strengths of each imaging modality has provided the ability to print morphologically accurate hybrid 3D models of cardiac structures [8]. The Radiological Society of North America 3D Printing Special Interest Group recently established appropriateness guidelines for 3D printing cardiac models including various other specific medical conditions. These guidelines include the best approach for acquisition of imaging, segmentation tools, 3D printing, and post-processing the models for CHD diagnosis and patient care [15]. 3D printing of CHD has been shown to be effective in double inlet left ventricle (DILV), double outlet left ventricle (DOLV), double outlet right ventricle (DORV), double inlet right ventricle (DIRV), congenitally corrected transposition of the great arteries (levo-TGA), transposition of the great arteries (dextro-TGA), unbalanced atrioventricular canal, Tetralogy of Fallot (ToF), truncus arteriosus, total anomalous pulmonary venous return (TAPVR) and partial anomalous pulmonary venous return (PAPVR) [15]. Cardiac models have proven valuable for pre-procedural planning, patient education, and training of medical professionals [16-22]. Research has provided insight into how valuable 3D printed models are with regards to postoperative care of CHD patients especially with patient hand-off versus a traditional verbal hand off [23]. Considering 3D printed heart models from CT or MRI have been accurate representations, yet there remains the unknown of whether the STL file and the 3D printed model are true representations in terms of measurements [24]. However, the heart is a dynamic organ 
and using static 3D models for cardiac visualization provides a limited representation of cardiac anatomy and physiology $[10,8,25]$. Further innovations in medical 3D printing include development of materials, which more accurately mimic cardiac tissues [26]. 3D bio-printing is another technology which may provide significant innovations in treatment of congenital and structural heart disease. The bioengineering of an electromechanically functional miniature ventricular heart chamber from human induced pluripotent stem cells has now been demonstrated [27,28]. Development of functional artificial chambers that can be safely implanted into patients will have a major impact on the treatment of complex CHD.

Interactive 3D Visualization. Major limitations of 3D imaging are the current display methods, i.e. displaying $3 \mathrm{D}$ imaging datasets on a $2 \mathrm{D}$ screen $[8,29]$. The introduction of augmented reality (AR) and virtual reality (VR) in medicine is another significant advancement with potential to significantly improve the care and management of patients. However, there is some skepticism with AR/VR systems, specifically the need to wear bulky or uncomfortable glasses/headsets. Some users may also experience motion sickness with the potential of compromising patient safety during procedures $[11,12]$. There are several companies working on solutions. One such solution is the True 3D Viewer (EchoPixel, Inc., Santa Clara, CA, USA) where imaging datasets sourced from CT, MR, and 3DE can be viewed in 3D space with the use of 3D glasses. Moreover, the system offers the user an interactive platform with the ability to rotate the images in multiple planes, perform dissections, segment the dataset, and perform measurements [30]. While the EchoPixel screen displays the image, lightweight 3D glasses with sensors are followed by tracking built into the display allowing the image to move with the viewer. This allows the now $3 \mathrm{D}$ image to be in perspective with the viewer and a stylus allows the user to manipulate the image and understand the complexity of the CHD. There are six viewing modes with each 
providing a different function allowing for images to be differentially analyzed (intuitive 2D, True 3D, C-arm Slab, Haptic annotation, radiology, and surgery). For CHD cases, having multiple viewing planes is particularly useful allowing for the heart to be evaluated from different perspectives. This technology is able to interpret and display CT, MR and XA standard DICOM/DICOMDIR files along with certain types of DICOM ultrasounds (GE vivid E90, E95 and Philips E33, GI, 3DDCM) [31]. EchoPixel has a toolset with several functions which include making measurements (including surface and volume), region growing segmentation, region of interest (ROI) selection, volume editing, and transfer function presets. These allow for CHD defects to be measured and analyzed as well as be prepared as a model for 3D printing within EchoPixel. These optimized image datasets can then be saved and referenced for later display for surgical or catheterization planning [31]. The segmentation tool allows for parts of the image to be transformed, visualized and potentially 3D printed. The C-arm view allows physicians to better view a fluoroscopy image by showing the orientation within the patient [32]. The bookmark tool helps with planning and actuating of surgeries wherein the surgeon bookmarks steps which allows for scrolling through during surgery creating a set of instructions with images for the procedure [33]. When paired with a 3D projector, the True 3D Viewer can be used with groups for pre-procedural planning, patient/family education, and training in multiple medical disciplines with clear resolution and depth perception (Figure $3 \& 4$; Supplementary Material 2 \& 3). 4D ultrasound datasets can also be viewed on True 3D Viewer with further development focused on visualization of 4D MR and integrated multimodality imaging.

Clinical utilization of EchoPixel has been found to improve surgical procedures in patients with CHD. In one study, approximately eighty percent of surgeons who looked at both EchoPixel and standard methods of imaging found it to be useful [34]. EchoPixel has been found to alter 
surgical techniques and repair strategies and offer additional insight in patients with borderline intracardiac anatomy for biventricular repair [35]. In a case at Stanford Children's Health, EchoPixel was used in the operating room to decrease the size of the surgical incision, which reduced recovery time and risk of infection for post-operative patients [35]. In another study, EchoPixel was used to measure the internal carotid artery length [36]. This measurement was taken several times on two patients and determined to be reproducible, showing the accuracy of the technology.

Real View Medical Holography (RealView Imaging Ltd., Israel) has designed a system, which uses holograph technology to display dynamic images, live holography, without requiring the user to wear a headset [37]. This has now been proven to be useful in the catheterization lab to show real time medical holograms - https://youtu.be/KLQCbDbljik [10]. In September 2018, the U.S. Food and Drug Administration granted NOVARAD (Salt Lake City, UT), 510(k) Clearance for use of their OpenSight AR system. This system allows the user to view AR in 2D, 3D and 4D simultaneously reducing the risk of disorientation [38]. The system supports preoperative planning providing the surgeon or interventionalist the advantage of comprehensively understanding the anatomy prior to performing a procedure. This technology has the possibility of decreasing procedure time and potential errors while improving outcomes. A recent study in eight patients undergoing cardiac catheterization procedures where RealView hologram was utilized along with standard imaging demonstrated the value of this product. The observers rated the hologram usefulness on a scale of 1-5 and all of them found it to be the most useful. Additionally none of them became nauseous, which is a common issue when utilizing augmented reality [10]. 
Artificial Intelligence. Recently, algorithms have been developed wherein computers may aid in heart segmentation in preparation for visualization or 3D printing [39]. Efficient segmentation methods may make it possible for scaling volumes of patients or urgent cases. Patch based interactive segmentation in which an operator manually segments a few anatomic slices while an algorithm completes the rest of the dataset. Current algorithms can create accurate models using only 14 segmented anatomic slices. This is significantly less than the normal process to create segmented models. Improving these algorithms may speed up this process, eventually allow for 3D printing to be utilized commonly in clinics [39]. Another version of this patch-based segmentation is being used for mitral valve visualization. This algorithm uses scans and coordinates as well as a moldable model to create a fitted model. This works well for a small region of interest, however has not been used for a whole heart or larger anatomic areas [40]. Another use of artificial intelligence is to compile patient data to determine what type of imaging to order and produce probable diagnoses. AI can also aid in deciding whether patients are eligible for a study. These processes are fully realized when large amounts of data are compiled and analyzed in one program. AI systems are also able to search the web or cloud-based systems and find cases from there. The difficulties of medical adoption of AI are programs which are fast and user friendly enough to be used in clinics [41]. Artificial neural networks (ANN) are the most common type of AI that are used in imaging. These systems use pathways which make connections and compare data. Along with this they weigh these connections to decide which path to go down or outcome to show. This process is developed to operate similarly to a neuron [42]. It can be "taught" but also learns from each diagnosis attempt. Through this learning process it can become increasingly accurate at choosing appropriate imaging or diagnosing 
patients. Considering AI and its application with CMR, challenges with AI and CMR with regards to CHD have been recognized and therefore need to be addressed moving forward [42].

\section{Future Advancements}

Future systems need to become more intuitive, allowing users the capability to perform tasks quickly, decreasing risks associated with inadequate preparation for procedures, and increase patient satisfaction and outcomes. Manufactures have yet to put a system on the market that allows for integrated multimodality imaging that can support dynamic 3D datasets. Medical imaging companies have been working in isolation with technology that supports AR and VR for various uses such as movies, flight simulation and other training systems. Some healthcare systems have been evaluating advanced imaging techniques on patients with cancer or other noncardiac medical diagnoses. The dynamic nature of the cardiac structures and the complexity of anomalies being limited to a vulnerable population of children and adults with CHD, results in significant limitations for the field of congenital cardiology. However, as precision medicine and corresponding treatment options grow, advanced imaging specialists are going to need this enhanced technology to help detect, diagnose, and guide procedures.

Clinical management of complex CHD patients often involves multiple imaging modalities to fully capture the complexity of patient anatomy. Individual imaging modalities are interpreted in isolation without integration on a common platform. Researchers and developers need to design a system with the ability to integrate the strengths of 3D imaging from CMR, CT, angiogram, 3D mapping, and 3DE with the ability to 3D print from them or display them in a dynamic format in a single platform. Moreover, developing a PACS capable of handling a large amount of data from multiple modalities would also be necessary. Current PACS may be capable; however, healthcare systems with PACS may have different software packages for various modalities 
using appropriate codecs. The next generation of imaging viewers must overcome these limitations. Future development of AI in the area of tissue characterization including incorporating echocardiogram dropouts with auto filling will improve the visualization of ultrasound-based 3D models.

Live imaging during the procedure could be sent directly to the AR system, and the interventionalist could perform the procedure without any fluoroscopy. This level of AR assisted procedures could reduce or eliminate radiation exposure and improve procedural outcomes.

Research and development of an AR system that does not require the user to wear a cumbersome headset, while allowing multiple viewers would be ideal for procedures in the operating room or catheterization laboratory. If AR imaging advances to this level, the need for pre-procedural tests may no longer be routinely required. Moreover, adding hemodynamic data to the integrated imaging datasets will improve assessment and interventions. The advantage of AR is its ability to integrate aspects of the virtual world with the real world, enhancing the objects that a person can feel, see, and hear. This would be an excellent tool for surgeons and interventionalists. Virtual interventional or surgical procedure using image fusion technology is within reach. Ultimately, this technology has the potential to benefit patients, improve surgical and interventional outcomes, reduce procedural errors and risks, and decrease costs associated with medical procedures and hospital admissions.

In addition to this, Artificial Intelligence (AI) is a field which has significant potential to aid imaging. Currently there are AI systems in development which may provide significant performance and be advantageous to CHD imaging. However, developing a system which can be used across a hospital and is very user friendly is not a reality yet. Creating a system which can perform the functions of storing and organizing patient data helping with computer aided 
diagnoses and other AI functions would be ideal. This would allow a smooth process for adoption of these new technologies. However, it is anticipated that implementing these systems in hospitals would take a significant amount of time.

\section{Conclusion}

Assessing current imaging systems and how innovative they have become leaves us to wonder what the future will hold. Since the beginning of medical imaging, technology has been pushing the medical visualization boundaries and enhanced the capabilities and medical professionals. Current systems are providing medical professionals with advanced imaging datasets to perform what is necessary to improve patient care, reduce surgical errors, and to preplan surgical or interventional procedures. These technological advances have made it possible for physicians to better appreciate the complexity of CHD and cardiac structures. Advances in integrated multimodality imaging is expected to democratize data interpretation from highly trained professionals of individual modalities to those directly interacting with patients. Advanced 3D imaging, and visualization of dynamic structures, 3D printing, and image manipulation are paving the way for research and development of integrated imaging systems utilizing artificial intelligence that will be able to perform far beyond what is currently available. 


\section{Compliance with Ethical Standards}

Conflict of interest: None.

Research involving human participants and/or animals: N/A

Informed consent: N/A 


\section{References}

1. Bradley WG (2008) History of medical imaging. Proc Am Philos Soc 152:349-361

2. Sundaram M, McGuire MH, Herbold DR (1988) Magnetic resonance imaging of soft tissue masses: an evaluation of fifty-three histologically proven tumors. Magn Reson Imaging 6:237248

3. Goitein O, Salem Y, Jacobson J, Goitein D, Mishali D, Hamdan A, Kuperstein R, Di Segni E, Konen E (2014) The role of cardiac computed tomography in infants with congenital heart disease. Isr Med Assoc J 16:147-152

4. Luijnenburg SE, Robbers-Visser D, Moelker A, Vliegen HW, Mulder BJ, Helbing WA (2010) Intra-observer and interobserver variability of biventricular function, volumes and mass in patients with congenital heart disease measured by CMR imaging. Int J Cardiovasc Imaging 26:57-64. doi:10.1007/s10554-009-9501-y

5. Black D, Vettukattil J (2013) Advanced echocardiographic imaging of the congenitally malformed heart. Curr Cardiol Rev 9:241-252

6. Samuel BP, Pinto C, Pietila T, Vettukattil JJ (2015) Ultrasound-Derived Three-Dimensional Printing in Congenital Heart Disease. J Digit Imaging 28:459-461. doi:10.1007/s10278-014$9761-5$

7. Gosnell J, Pietila T, Samuel BP, Kurup HK, Haw MP, Vettukattil JJ (2016) Integration of Computed Tomography and Three-Dimensional Echocardiography for Hybrid ThreeDimensional Printing in Congenital Heart Disease. J Digit Imaging 29:665-669. doi:10.1007/s10278-016-9879-8 
8. Kurup HK, Samuel BP, Vettukattil JJ (2015) Hybrid 3D printing: a game-changer in personalized cardiac medicine? Expert Rev Cardiovasc Ther 13:1281-1284. doi:10.1586/14779072.2015.1100076

9. Wang KC, Filice RW, Philbin JF, Siegel EL, Nagy PG (2011) Five levels of PACS modularity: integrating 3D and other advanced visualization tools. J Digit Imaging 24:10961102. doi:10.1007/s10278-011-9366-1

10. Bruckheimer E, Rotschild C, Dagan T, Amir G, Kaufman A, Gelman S, Birk E (2016) Computer-generated real-time digital holography: first time use in clinical medical imaging. Eur Heart J Cardiovasc Imaging 17:845-849. doi:10.1093/ehjci/jew087

11. Glatz AC, Purrington KS, Klinger A, King AR, Hellinger J, Zhu X, Gruber SB, Gruber PJ (2014) Cumulative exposure to medical radiation for children requiring surgery for congenital heart disease. J Pediatr 164:789-794.e710. doi:10.1016/j.jpeds.2013.10.074

12. Hoffmann A, Engelfriet P, Mulder B (2007) Radiation exposure during follow-up of adults with congenital heart disease. Int J Cardiol 118:151-153. doi:10.1016/j.ijcard.2006.07.012 13. Farooqi KM, Sengupta PP (2015) Echocardiography and three-dimensional printing: sound ideas to touch a heart. J Am Soc Echocardiogr 28:398-403. doi:10.1016/j.echo.2015.02.005 14. Vettukattil JJSBPGJMKHKN (2017) Creation of a 3D Printed Model: From Virtual to Physical. Rapid Prototyping in Cardiac Disease, 1 edn. Springer International Publishing AG. doi:10.1007/978-3-319-53523-4_2

15. Chepelev L, Wake N, Ryan J, Althobaity W, Gupta A, Arribas E, Santiago L, Ballard DH, Wang KC, Weadock W, Ionita CN, Mitsouras D, Morris J, Matsumoto J, Christensen A, Liacouras P, Rybicki FJ, Sheikh A, Printing RSIGfD (2018) Radiological Society of North 
America (RSNA) 3D printing Special Interest Group (SIG): guidelines for medical 3D printing and appropriateness for clinical scenarios. 3D Print Med 4:11. doi:10.1186/s41205-018-0030-y 16. Costello JP, Olivieri LJ, Su L, Krieger A, Alfares F, Thabit O, Marshall MB, Yoo SJ, Kim PC, Jonas RA, Nath DS (2015) Incorporating three-dimensional printing into a simulation-based congenital heart disease and critical care training curriculum for resident physicians. Congenit Heart Dis 10:185-190. doi:10.1111/chd.12238

17. Kim MS, Hansgen AR, Wink O, Quaife RA, Carroll JD (2008) Rapid prototyping: a new tool in understanding and treating structural heart disease. Circulation 117:2388-2394. doi:10.1161/CIRCULATIONAHA.107.740977

18. Olivieri L, Krieger A, Chen MY, Kim P, Kanter JP (2014) 3D heart model guides complex stent angioplasty of pulmonary venous baffle obstruction in a Mustard repair of D-TGA. Int J Cardiol 172:e297-298. doi:10.1016/j.ijcard.2013.12.192

19. Schmauss D, Gerber N, Sodian R (2013) Three-dimensional printing of models for surgical planning in patients with primary cardiac tumors. J Thorac Cardiovasc Surg 145:1407-1408. doi:10.1016/j.jtcvs.2012.12.030

20. Schmauss D, Schmitz C, Bigdeli AK, Weber S, Gerber N, Beiras-Fernandez A, Schwarz F, Becker C, Kupatt C, Sodian R (2012) Three-dimensional printing of models for preoperative planning and simulation of transcatheter valve replacement. Ann Thorac Surg 93:e31-33. doi:10.1016/j.athoracsur.2011.09.031

21. Sodian R, Weber S, Markert M, Loeff M, Lueth T, Weis FC, Daebritz S, Malec E, Schmitz C, Reichart B (2008) Pediatric cardiac transplantation: three-dimensional printing of anatomic models for surgical planning of heart transplantation in patients with univentricular heart. $\mathbf{J}$ Thorac Cardiovasc Surg 136:1098-1099. doi:10.1016/j.jtcvs.2008.03.055 
22. Vettukattil JJ, Mohammad Nijres B, Gosnell JM, Samuel BP, Haw MP (2019) Threedimensional printing for surgical planning in complex congenital heart disease. J Card Surg 34:1363-1369. doi:10.1111/jocs.14180

23. Sun Z, Lau I, Wong YH, Yeong CH (2019) Personalized Three-Dimensional Printed Models in Congenital Heart Disease. J Clin Med 8. doi:10.3390/jcm8040522

24. Arafati A, Hu P, Finn JP, Rickers C, Cheng AL, Jafarkhani H, Kheradvar A (2019) Artificial intelligence in pediatric and adult congenital cardiac MRI: an unmet clinical need. Cardiovasc Diagn Ther 9:S310-S325. doi:10.21037/cdt.2019.06.09

25. O'Neill B, Wang DD, Pantelic M, Song T, Guerrero M, Greenbaum A, O'Neill WW (2015) Reply: The Role of 3D Printing in Structural Heart Disease: All That Glitters Is Not Gold. JACC Cardiovasc Imaging 8:988-989. doi:10.1016/j.jcmg.2015.04.011

26. Anwar S, Singh GK, Miller J, Sharma M, Manning P, Billadello JJ, Eghtesady P, Woodard PK (2018) 3D Printing is a Transformative Technology in Congenital Heart Disease. JACC Basic Transl Sci 3:294-312. doi:10.1016/j.jacbts.2017.10.003

27. Li RA, Keung W, Cashman TJ, Backeris PC, Johnson BV, Bardot ES, Wong AOT, Chan PKW, Chan CWY, Costa KD (2018) Bioengineering an electro-mechanically functional miniature ventricular heart chamber from human pluripotent stem cells. Biomaterials 163:116127. doi:10.1016/j.biomaterials.2018.02.024

28. Lee A, Hudson AR, Shiwarski DJ, Tashman JW, Hinton TJ, Yerneni S, Bliley JM, Campbell PG, Feinberg AW (2019) 3D bioprinting of collagen to rebuild components of the human heart. Science (New York, NY) 365:482-487. doi:10.1126/science.aav9051

29. Simpson JM (2016) Three-dimensional echocardiography in congenital heart disease: The next steps. Arch Cardiovasc Dis 109:81-83. doi:10.1016/j.acvd.2015.09.010 
30. Ballocca F, Meier LM, Ladha K, Qua Hiansen J, Horlick EM, Meineri M (2019) Validation of Quantitative 3-Dimensional Transesophageal Echocardiography Mitral Valve Analysis Using Stereoscopic Display. J Cardiothorac Vasc Anesth 33:732-741. doi:10.1053/j.jvca.2018.08.013 31. True 3D Viewer User Manual (2018). L edn. EchoPixel, Inc., Santa Clara, CA

32. EchoPixel showcases next-generation surgical planning with True 3-D interactive mixed reality software (2018). Latest version of True 3-D expands supported modalities beyond CT and MR to include ultrasound with Doppler and C-arm views. Imaging Technology News (ITN), 33. EchoPixel announces True 3-D print support (2017). Suite of software tools built on EchoPixel's interactive virtual reality technology aim to provide fast, accurate, 3-D models for a wide range of medical procedures. Imaging Technology News (ITN),

34. Lu J, Ensing G, Ohye R, Romano J, Sassalos P (2019) Virtual reality three-dimensional modeling for congenital heart surgery planning. American Society of Echocardiography (ASE) 2019 ,

35. Haw M, Baliulis G, Hillman N, Samuel B, Gosnell J, Byl J, Vettukattil J (2019) 3D printing and interactive visualization for surgical planning in complex congenital heart disease.

Congenital Heart Surgeons' Society (CHSS) 2019,

36. I K, M A, I C (2016) E-010 Vessel length spline measurement with EchoPixel True 3D Viewer. vol 8. J NeuroInterv Surg,

37. Mishra S (2017) Hologram the future of medicine - From Star Wars to clinical imaging. Indian Heart J 69:566-567. doi:10.1016/j.ihj.2017.07.017

38. FDA US (2018) 510(k) Premarket Approval. 
39. Pace DF, Dalca AV, Geva T, Powell AJ, Moghari MH, Golland P (2015) Interactive WholeHeart Segmentation in Congenital Heart Disease. Med Image Comput Comput Assist Interv 9351:80-88. doi:10.1007/978-3-319-24574-4_10

40. Pouch AM, Wang H, Takabe M, Jackson BM, Gorman JH, Gorman RC, Yushkevich PA, Sehgal CM (2014) Fully automatic segmentation of the mitral leaflets in 3D transesophageal echocardiographic images using multi-atlas joint label fusion and deformable medial modeling. Med Image Anal 18:118-129. doi:10.1016/j.media.2013.10.001

41. Dilsizian SE, Siegel EL (2014) Artificial intelligence in medicine and cardiac imaging: harnessing big data and advanced computing to provide personalized medical diagnosis and treatment. Curr Cardiol Rep 16:441. doi:10.1007/s11886-013-0441-8

42. Fazal MI, Patel ME, Tye J, Gupta Y (2018) The past, present and future role of artificial intelligence in imaging. Eur J Radiol 105:246-250. doi:10.1016/j.ejrad.2018.06.020 


\section{Figure legend}

Figure 1. Automated and semi-automated segmentation of CT on Mimics Innovation Suite (Materialise, N.V., Leuven, Belgium) depicting the coronal, axial, and sagittal views of the CT, and the segmented virtual model.

Figure 2. The sagittal view of the segmented virtual model shows the compressed pulmonary artery conduit (red arrows) between the sternum and the myocardium.

Supplementary Material 1. 3D segmented virtual model.

Figure 3A. CT viewed on True 3D Viewer (EchoPixel, Inc., Santa Clara, CA, USA).

Figure 3B. CT viewed on True 3D Viewer with integration of segmented 3D heart model (EchoPixel, Inc., Santa Clara, CA, USA).

Figure 4A. 3D glasses and stylus are used to interact with the imaging datasets on True 3D Viewer (EchoPixel, Inc., Santa Clara, CA, USA).

Figure 4B. Use of stylus is shown on True 3D Viewer (EchoPixel, Inc., Santa Clara, CA, USA).

Supplementary Material 2. A $360^{\circ}$ visualization in any plane is feasible on True 3D Viewer (EchoPixel, Inc., Santa Clara, CA, USA).

Supplementary Material 3. A $360^{\circ}$ visualization in any plane is feasible on True 3D Viewer (EchoPixel, Inc., Santa Clara, CA, USA). 

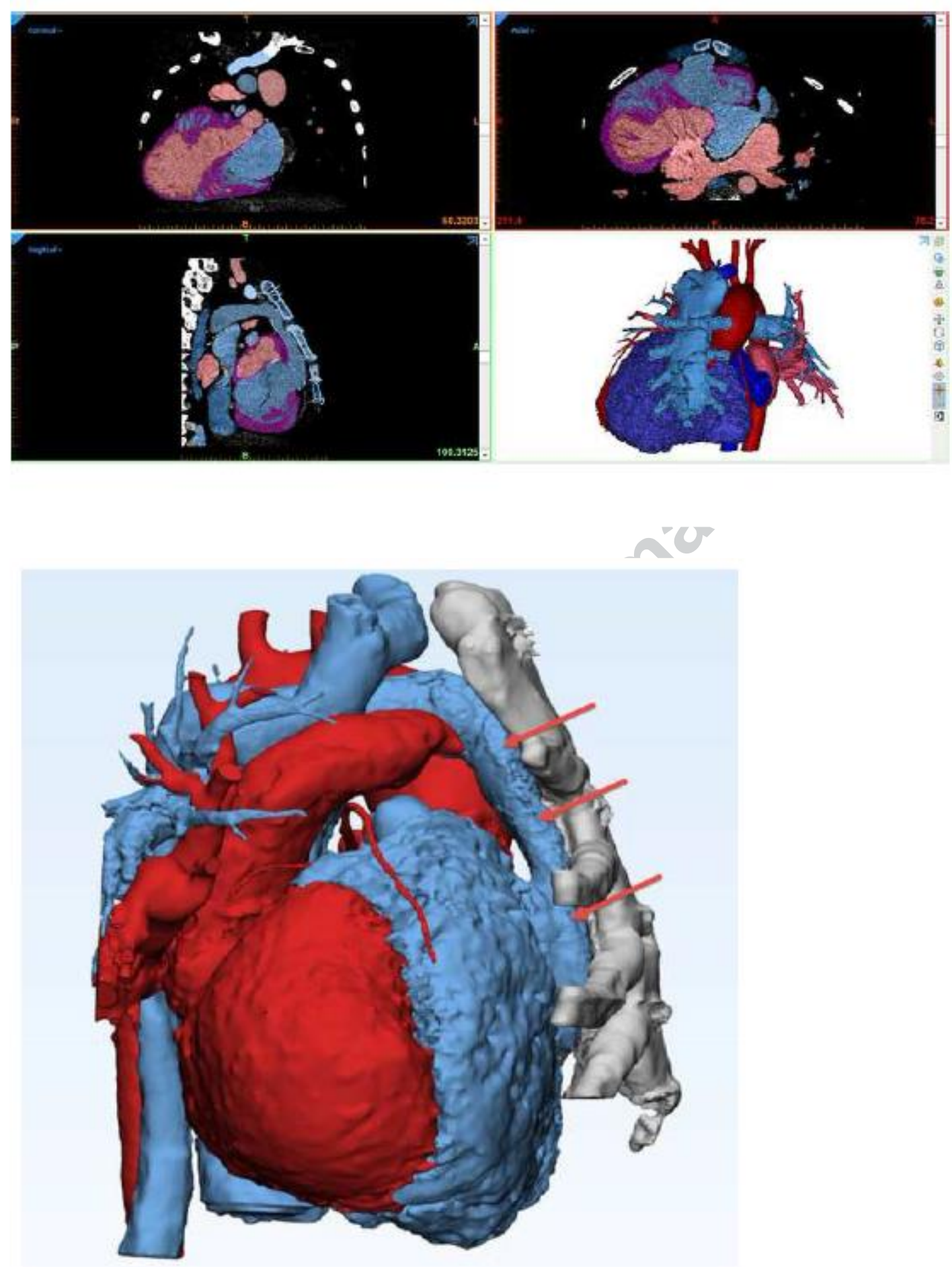

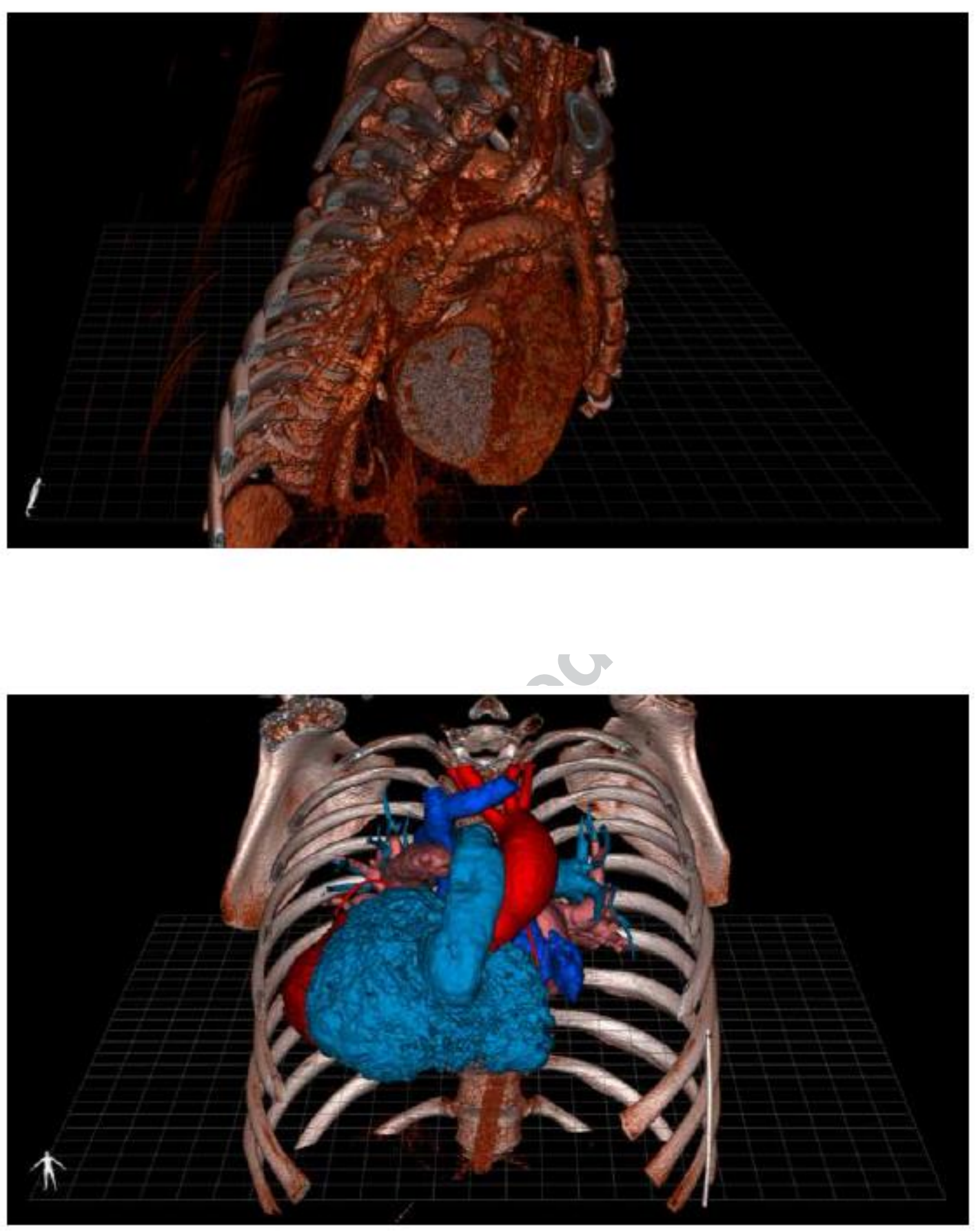

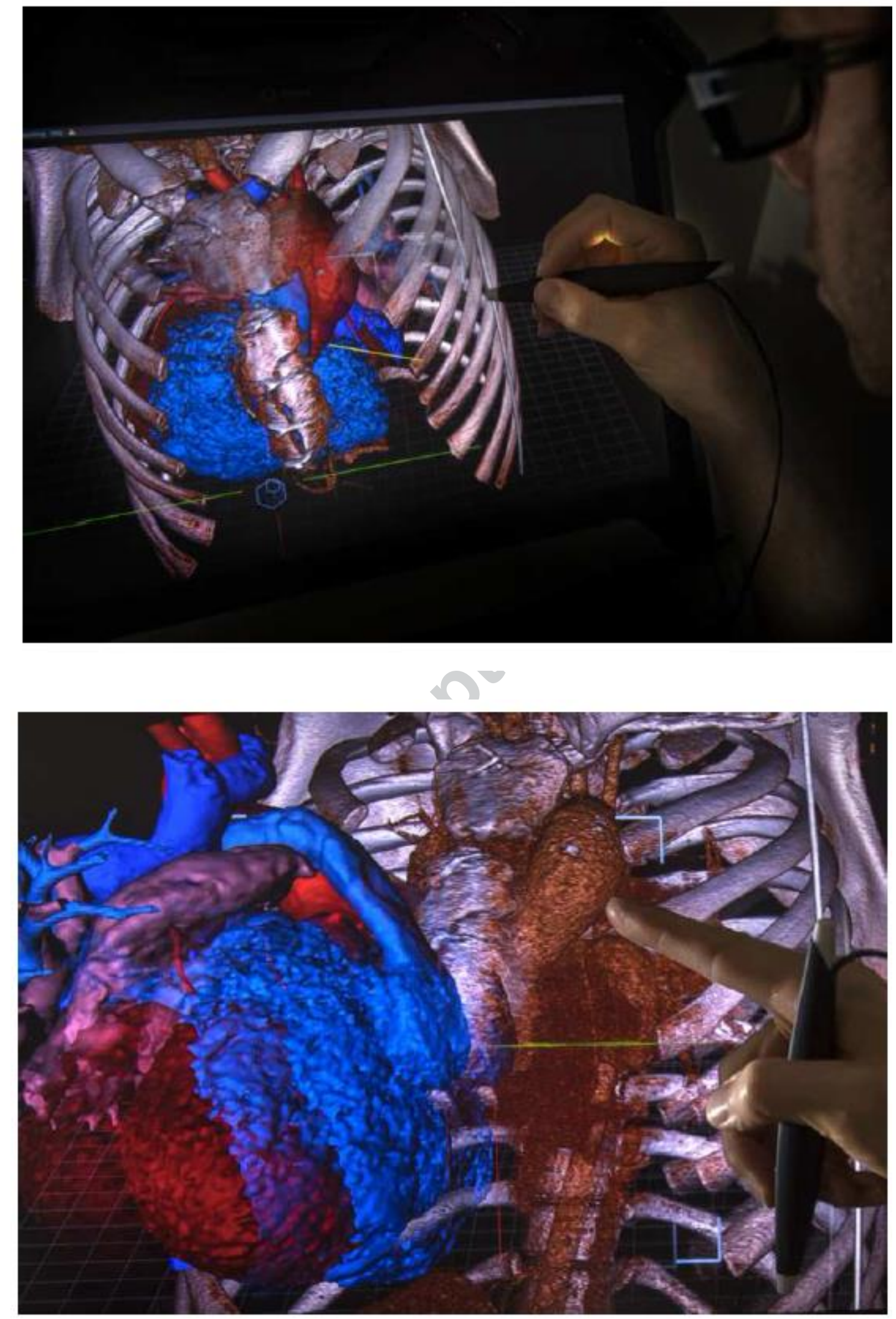